\title{
GENERALIZED REGULAR VARIATION OF SECOND ORDER
}

\author{
LAURENS DE HAAN and ULRICH STADTMÜLLER
}

(Received 20 March 1994; revised 11 December 1995)

Communicated by R. A. Maller

\begin{abstract}
Assume that for a measurable function $f$ on $(0, \infty)$ there exist a positive auxiliary function $a(t)$ and some $\gamma \in \mathbb{R}$ such that $\phi(x)=\lim _{t \rightarrow \infty}(f(t x)-f(t)) / a(t)=\int_{1}^{x} s^{\gamma-1} d s, x>0$. Then $f$ is said to be of generalized regular variation. In order to control the asymptotic behaviour of certain estimators for distributions in extreme value theory we are led to study regular variation of second order, that is, we assume that $\lim _{t \rightarrow \infty}(f(t x)-f(t)-a(t) \phi(x)) / a_{1}(t)$ exists non-trivially with a second auxiliary function $a_{1}(t)$. We study the possible limit functions in this limit relation (defining generalized regular variation of second order) and their domains of attraction. Furthermore we give the corresponding relation for the inverse function of a monotone $f$ with the stated property. Finally, we present an Abel-Tauber theorem relating these functions and their Laplace transforms.
\end{abstract}

1991 Mathematics subject classification (Amer. Math. Soc.): 26A12, 40E05.

Keywords and phrases: Regular variation, second order variation, limit functions, domain of attraction, inverse functions, Laplace transform, Abelian theorem, Tauberian theorem.

\section{Introduction}

Throughout we consider measurable real-valued functions on $(0, \infty)$ which are bounded on $(0, a)$ for all $a>0$. The class of regularly varying functions, that is, eventually positive functions, satisfying

$$
\lim _{t \rightarrow \infty} \frac{f(t x)}{f(t)} \text { exists and is positive for all } x>0,
$$

plays an important role in the asymptotic analysis of various problems. It is well known that (1.1) is equivalent to

(1.1') $\lim _{t \rightarrow \infty} \frac{f(t x)}{f(t)}=x^{\alpha}, \quad$ for $x>0$, with some $\alpha \in \mathbb{R} \quad$ (notation: $f \in R V_{\alpha}$ )

(c) 1996 Australian Mathematical Society $0263-6115 / 96 \$ A 2.00+0.00$ 
(consult, for example, any of the books $[1,8,15]$ ). The number $\alpha$ is called the index of regular variation. In extreme value theory and associated statistics one is interested in functions $f(\cdot)$ having the following property:

Assume that there exists some positive function $a(\cdot)$ such that

$$
\lim _{t \rightarrow \infty} \frac{f(t x)-f(t)}{a(t)} \quad \text { exists for all } x>0 .
$$

A distribution function $F$ is in the domain of attraction of an extreme value distribution if and only if (1.2) holds for the inverse function of $(1 /(1-F(\cdot)))$ with limit $\not \equiv 0$.

The following results, connecting (1.2) with (1.1), respectively $\left(1.1^{\prime}\right)$, are well known (see, for example, [8]).

PROPOSITION 1. If (1.2) holds, then we have

(i) The limit function in (1.2) is given by $c_{0} \int_{1}^{x} s^{\gamma-1} d s$ with some $c_{0}, \gamma \in \mathbb{R}$;

(ii) If $c_{0} \neq 0$, then the auxiliary function $a(\cdot)$ is regularly varying with index $\gamma$; that is $a(\cdot) \in R V_{\gamma}$.

REMARKS 1. (i) We write $\left(x^{\gamma}-1\right) / \gamma$ for $\int_{1}^{x} s^{\gamma-1} d s$, even in case $\gamma=0$ where it is $\log x$.

(ii) The interesting case is when $c_{0} \neq 0$ and without loss of generality we may assume $c_{0}>0$ (otherwise consider $-f$ ). But then we may write for (1.2)

$$
\lim _{t \rightarrow \infty} \frac{f(t x)-f(t)}{a(t)}=\frac{x^{\gamma}-1}{\gamma}, \quad \text { for } x>0
$$

with a (possibly modified) auxiliary function $a(\cdot)$ and some constant $\gamma \in \mathbb{R}$.

From $[8$, Theorem 1.10$]$ or $[1$, section 3.2$]$ we obtain

PROPOSITION 2. Suppose that $f$ satisfies $\left(1.2^{\prime}\right)$ with some auxiliary function $a(\cdot)>0$.

(i) If $\gamma>0$ then $f$ is eventually positive and $f \in R V_{\gamma}$, that is,

$$
\lim _{t \rightarrow \infty} \frac{f(t x)}{f(t)}=x^{\gamma}, \quad \text { for } \quad x>0 .
$$

(ii) If $\gamma<0$, then $\lim _{t \rightarrow \infty} f(t)=: f(\infty)$ exists and $f(\infty)-f(t) \in R V_{\gamma}$, that is,

$$
\lim _{t \rightarrow \infty} \frac{f(\infty)-f(t x)}{f(\infty)-f(t)}=x^{\gamma}, \quad \text { for } \quad x>0 .
$$


REMARKS 2. (i) Note that (1.3) respectively (1.4) imply $\left(1.2^{\prime}\right)$ with a suitable choice of $a(\cdot)$. Take, for example, $a(t)=\gamma f(t)$ in case (1.3) and $a(t)=-\gamma(f(\infty)-f(t))$ in case (1.4).

(ii) A function $f(\cdot)$ satisfying $\left(1.2^{\prime}\right)$ with $\gamma=0$ is said to be $\Pi$-varying (at infinity) and we write $f \in \Pi$. For further information about this function class consult, for example, [8, sect. I.2], or [1, chap. 3].

For statistics in extreme value theory, for example, to estimate the parameter $\gamma$ in regularly varying tails of distribution functions (see, for example, [3, Lemma 2.2 ] and the subsection below) one uses an empirical version of $\left(1.2^{\prime}\right)$. In order to get information about the speed of convergence of these estimators one needs among other things second-order properties of the function $f$. This has been done in connection with statistical problems for very special second-order behaviour (see, for example, [3], Theorems 2.3 and 2.5 , cf. Remarks 2.2 and 2.3 and the references therein). In order to get our hands on the most general case, we may proceed as follows (see also [4]).

Let be given a function $f(\cdot)$ with auxiliary function $a(\cdot)$ satisfying the asymptotic relation $\left(1.2^{\prime}\right)$. Now assume furthermore that there exists some positive function $A(\cdot)$ with $\lim _{t \rightarrow \infty} A(t)=3$, such that

(1.5) $\lim _{t \rightarrow \infty}\left\{\frac{f(t x)-f(t)}{a(t)}-\frac{x^{\gamma}-1}{\gamma}\right\} / A(t)$ exists non-trivially on $(0, \infty)$.

What we mean by 'non-trivially' will be explained shortly. Clearly the function $A(t)$ describes the rate of convergence in $\left(1.2^{\prime}\right)$. Denoting $a_{1}(t):=a(t) A(t)=o(a(t))$, $t \rightarrow \infty$, we write

$$
H(x)=\lim _{t \rightarrow \infty} \frac{f(t x)-f(t)-a(t)\left(x^{\gamma}-1\right) / \gamma}{a_{1}(t)}, \quad x>0 .
$$

We want to find the possible limit functions $H$ in (1.6) and the choices of the auxiliary functions $a_{1}(\cdot)$ which may occur in (1.6). Note that by adding $c \cdot a_{1}(t)$ to $a(t)$ in (1.6) we get the same relation with a new function $\tilde{a}($.$) and a new limit function \tilde{H}($.$) and$ we have

$$
a(t) \sim \tilde{a}(t), \quad t \rightarrow \infty \quad \text { and } \quad \tilde{H}(x)=H(x)-c \frac{x^{\gamma}-1}{\gamma}
$$

This means that we can always add a multiple of $\left(x^{\gamma}-1\right) / \gamma$ to the function $H(x)$. So $H(x)$ is determined up to a multiple of $\left(x^{\gamma}-1\right) / \gamma$ only. Observe that replacing $a(\cdot)$ by $\tilde{a}(\cdot)$ does not change $\left(1.2^{\prime}\right)$. Hence a non-trivial limit in (1.5) is a function $\mathrm{H}$ which is not a multiple of $\left(x^{\gamma}-1\right) / \gamma$; in particular, $H$ is not identically zero. 
Applications of second order variation Second order variation plays an important role in extreme value theory. For a simple example consider i.i.d. random variables $X_{1}, X_{2}, \ldots$ from some distribution function $F$ and suppose that $1-F \in R V_{-\rho}$ for some $\rho>0$. A well-known estimator for $\gamma:=1 / \rho$ is given by

$$
\hat{\gamma}_{n}:=\frac{1}{k} \sum_{j=0}^{k-1} \log X_{(n-j, n)}-\log X_{(n-k, n)}
$$

where $k \leq n$ and $\left\{X_{(j, n)}\right\}$ denotes the $n$th order statistics of $X_{1}, \ldots, X_{n}$. It is well known (cf. [2, Theorem 4.1]) that for such a distribution $F$ we have that

$$
\sqrt{k}\left(1-\hat{\gamma}_{n} / \gamma_{n}\right) \text { is asymptotically normal, }
$$

provided that $k=k(n) \rightarrow \infty, k(n) / n \rightarrow 0(n \rightarrow \infty)$ and $\gamma_{n}$ is defined by

$$
\gamma_{n}:=\frac{n}{k} \int_{U(n / k)}^{\infty} \log s d F(s)
$$

where the function $U$ is the inverse of $1 /(1-F)$. Now note that under the stated conditions $\lim _{n \rightarrow \infty} \gamma_{n}=\gamma$. If one wants a confidence interval for $\gamma$ based on $\hat{\gamma}_{n}$, one needs the asymptotic normality of $\sqrt{k}\left(1-\hat{\gamma}_{n} / \gamma\right)$. This follows from (1.8) provided

$$
\lim _{n \rightarrow \infty} \sqrt{k}\left(\gamma_{n}-\gamma\right)=0
$$

Here the second-order condition comes in, as we shall show. We can write

$$
\gamma_{n}=\frac{n}{k} \int_{\frac{n}{k}}^{\infty} \frac{d(\log U(s))}{s}=\int_{1}^{\infty}\left\{\log U\left(\frac{n}{k} v\right)-\log U\left(\frac{n}{k}\right)\right\} \frac{d v}{v^{2}}
$$

Hence

$$
\sqrt{k}\left(\gamma_{n}-\gamma\right)=\int_{1}^{\infty} \sqrt{k}\left\{\log U\left(\frac{n}{k} v\right)-\log U\left(\frac{n}{k}\right)-\gamma \log v\right\} \frac{d v}{v^{2}} .
$$

The integral converges to zero if

$$
\lim _{n \rightarrow \infty} \sqrt{k}\left\{\log U\left(\frac{n}{k} v\right)-\log U\left(\frac{n}{k}\right)-\gamma \log v\right\}=0
$$

uniformly for $v \geq 1$. This condition imposes a restriction both on $U$ and on the sequence $k(n)$. In order to separate out the two conditions we assume that there exists a positive function $A$ such that

$$
\lim _{t \rightarrow \infty} \frac{\log U(t x)-\log U(t)-\gamma \log x}{A(t)}=: H(x)
$$


exists for $x>0$ and that the sequence $k(n)$ satisfies $\lim _{n \rightarrow \infty} \sqrt{k(n)} A(n / k(n))=0$. Then (1.9) follows. Note that (1.10) is a special instance of (1.6).

If one considers domains of attraction of all the extreme value distributions $\left\{G_{\gamma}\right\}_{\gamma \in \mathbb{B}}$ and not just the case $\gamma>0$ as above, one is led to the full generality of (1.6) (cf. Dekkers and de Haan [3]).

Another use of second-order conditions in extreme value theory involves finding the optimal choice of the sequence $k(n)$ mentioned above, in order to make $E\left(\gamma_{n}-\gamma\right)^{2}$ minimal (cf. Hall [12] and Dekkers and de Haan [4]).

Finally, second-order conditions are used to establish a rate of convergence of the distribution of sample maxima towards an extreme-value distribution (Smith [16], De Haan and Resnick [11]). Further results about second-order conditions can be found in $[5,9,14]$.

In Section 2 we shall characterize the limit functions $H(\cdot)$ and their domain of attraction. In Section 3 we consider the corresponding relations for the inverse function of a monotone function $f$. Finally (in Section 4) we discuss how (1.6) is reflected in the asymptotic behaviour of the Laplace transform of $f$.

\section{Limit functions and domain of attraction}

The limit functions $H$ are characterized in the following result.

THEOREM 1. Let be given a function $f$. Suppose that there exist $\gamma \in \mathbb{R}$ and positive auxiliary functions $a(\cdot)$ and $a_{1}(\cdot)$ such that the limit in (1.6) exists for all $x>0$. If $H$ is not a multiple of $\left(x^{\gamma}-1\right) / \gamma$, then there exist constants $c_{1}, c_{2} \in \mathbb{R}$ and some parameter $\alpha \leq 0$ such that:

$$
H(x)=c_{1} \int_{1}^{x} s^{\gamma-1} \int_{1}^{s} u^{\alpha-1} d u d s+c_{2} \int_{1}^{x} s^{\alpha+\gamma-1} d s, \quad x>0 .
$$

Moreover for $x>\mathrm{C}$

$$
\lim _{t \rightarrow \infty} \frac{(t x)^{-\gamma} a(t x)-t^{-\gamma} a(t)}{t^{-\gamma} a_{1}(t)}=c_{1} \frac{x^{\alpha}-1}{\alpha}
$$

and

$$
\lim _{t \rightarrow \infty} \frac{a_{1}(t x)}{a_{1}(t)}=x^{\alpha+\gamma}
$$


PROOF. Consider for $x, y>0$ the identity

$$
\begin{aligned}
x^{-\gamma}\{ & \left.\frac{f(t x y)-f(t)-a(t)\left((x y)^{\gamma}-1\right) / \gamma}{a_{1}(t)}-\frac{f(t x)-f(t)-a(t)\left(x^{\gamma}-1\right) / \gamma}{a_{1}(t)}\right\} \\
= & \frac{f(t x y)-f(t x)-a(t x)\left(y^{\gamma}-1\right) / \gamma}{a_{1}(t x)} \cdot \frac{(t x)^{-\gamma} a_{1}(t x)}{t^{-\gamma} a_{1}(t)} \\
& +\frac{y^{\gamma}-1}{\gamma} \cdot \frac{(t x)^{-\gamma} a(t x)-t^{-\gamma} a(t)}{t^{-\gamma} a_{1}(t)} .
\end{aligned}
$$

Letting $t \rightarrow \infty$ on both sides we obtain by (1.5) and (1.6)

$$
x^{-\gamma}\{H(x y)-H(x)\}
$$

$$
=\lim _{t \rightarrow \infty}\left\{H(y)(1+o(1)) \frac{(t x)^{-\gamma} a_{1}(t x)}{t^{-\gamma} a_{1}(t)}+\frac{y^{\gamma}-1}{\gamma} \frac{(t x)^{-\gamma} a(t x)-t^{-\gamma} a(t)}{t^{-\gamma} a_{1}(t)}\right\} .
$$

By assumption, there exist $y_{1}, y_{2} \in \mathbb{R}$ such that $\left(H\left(y_{1}\right),\left(y_{1}^{\gamma}-1\right) / \gamma\right)$ and $\left(H\left(y_{2}\right)\right.$, $\left.\left(y_{2}^{\gamma}-1\right) / \gamma\right)$ are linearly independent; hence with $\lambda=\left(y_{1}^{\gamma}-1\right) /\left(y_{2}^{\gamma}-1\right)$ we obtain that $H\left(y_{1}\right)-\lambda H\left(y_{2}\right) \neq 0$. Now we subtract $\lambda$ times (2.4) at argument $y=y_{2}$ from (2.4) with argument $y=y_{1}$ and we find, denoting by

$$
\psi\left(x, y_{1}, y_{2}\right):=x^{-\gamma}\left\{\left(H\left(x y_{1}\right)-H(x)\right)-\frac{y_{1}^{\gamma}-1}{y_{2}^{\gamma}-1}\left(H\left(x y_{2}\right)-H(x)\right)\right\},
$$

that

$$
\psi\left(x, y_{1}, y_{2}\right)=\lim _{t \rightarrow \infty}\left\{\left(H\left(y_{1}\right)-\lambda H\left(y_{2}\right)\right)(1+o(1)) \frac{(t x)^{-\gamma} a_{1}(t x)}{t^{-\gamma} a_{1}(t)}\right\}, x>0 .
$$

From this, we conclude that $\lim _{t \rightarrow \infty}(t x)^{-\gamma} a_{1}(t x) /\left(t^{-\gamma} a_{1}(t)\right)=: q(x)$ exists for all $x>0$. It is easy to see that either $q(x)$ is zero for all $x>0$, or positive for all $x>0$. The existence of this limit, together with relation (2.4), implies that $\lim _{t \rightarrow \infty}\left((t x)^{-\gamma} a(t x)-t^{-\gamma} a(t)\right) /\left(t^{-\gamma} a_{1}(t)\right)$ also exists for all $x>0$. Hence we obtain (2.2). If $q(x) \equiv 0$, the constant $c_{1}$ in (2.2) must be zero and it is easy to see that then $H(\cdot)$ is constant. Hence $c_{1} \neq 0$, and $q(x)$ is positive and thus (2.3) holds with some $\alpha \in \mathbb{R}$. By the arguments in (1.7) we can now assume again that $c_{1} \neq 0$. Then we must have $\alpha \leq 0$, since for $\alpha>0$ we would have $\lim _{t \rightarrow \infty} a(t x) / a(t)=x^{\alpha+\gamma}$ according to Proposition 2; but this is incompatible with Proposition 1(ii) applied to $f$, which states that $\lim _{t \rightarrow \infty} a(t x) / a(t)=x^{\gamma}$.

As a result, we obtain the following functional equation for $H$ :

$$
H(x \cdot y)=H(y) x^{\alpha+\gamma}+H(x)+c_{1} x^{\gamma} \frac{y^{\gamma}-1}{\gamma} \frac{x^{\alpha}-1}{\alpha}, \quad \text { for } x, y>0 .
$$


A simple calculation verifies that

$$
H_{1}(x)=c_{1} \int_{1}^{x} s^{\gamma-1} \int_{1}^{s} u^{\alpha-1} d u d s
$$

is a special solution of (2.6). Obviously, the function $G(x)=H(x)-H_{1}(x)$ satisfies the homogeneous equation

$$
G(x y)=G(x)+G(y) x^{\alpha+\gamma}, \quad \text { for } x, y>0 .
$$

If $\alpha+\gamma=0$, this is Cauchy's equation, having the only solution $G(x)=c_{1} \log x$ in the class of measurable functions. If $\alpha+\gamma \neq 0$, by symmetry we obtain:

$$
G(x y)=G(y)+G(x) y^{\alpha+\gamma}
$$

and hence $G(x)\left(1-y^{\alpha+\gamma}\right)=G(y)\left(1-x^{\alpha+\gamma}\right)$, for $x, y>0$, which implies that with some $c_{2} \in \mathbb{R}, G(x)=c_{2}\left(x^{\alpha+\gamma}-1\right) /(\alpha+\gamma)$, yielding the desired result.

REMARKS 3. (i) Observe that by the results above, the function $A(\cdot)$, describing the rate of convergence in (1.2), satisfies

$$
\lim _{t \rightarrow \infty} A(t x) / A(t)=x^{\alpha}, \quad x>0, \quad \text { for some } \alpha \leq 0 .
$$

So if $\alpha<0$ we have an algebraic speed of convergence in (1.2). In case $\alpha=0$ it is much slower, for example, logarithmic.

(ii) From (2.1) we see that $H(x)$ can be written as

$$
H(x)= \begin{cases}c_{1}(\log x)^{2} / 2+c_{2} \log x, & \text { if } \alpha=\gamma=0, \\ \frac{c_{1}}{\gamma} x^{\gamma} \log x+\left(c_{2}-c_{1} / \gamma\right) \frac{x^{\gamma}-1}{\gamma}, & \text { if } \alpha=0, \gamma \neq 0, \\ \left(\frac{c_{1}}{\alpha}+c_{2}\right) \frac{x^{\gamma+\alpha}-1}{\alpha+\gamma}-\frac{c_{1}}{\alpha} \frac{x^{\gamma}-1}{\gamma}, & \text { if } \alpha<0, \alpha+\gamma \neq 0, \\ \left(\frac{c_{1}}{\alpha}+c_{2}\right) \log x-\frac{c_{1}}{\alpha} \frac{x^{\gamma}-1}{\gamma}, & \text { if } \alpha<0, \alpha+\gamma=0 .\end{cases}
$$

Note that the last term in each case is a multiple of $\left(x^{\gamma}-1\right) / \gamma$; hence we must have $c_{1} \neq-\alpha c_{2}$.

Theorem 1 is the second-order analogue of Proposition 1. Next we shall give a result which corresponds to Proposition 2 . That is, we ask which functions $f$ do satisfy (1.6) with a limit function $H$ having certain parameters $\alpha, \gamma$. For $\alpha=\gamma=0$ the conditions have been given by Omey and Willekens [13]. Note that by $\pm h \in R V_{\delta}$ or $h \in \pm R V_{\delta}$ we mean $h \in R V_{\delta}$ or $-h \in R V_{\delta}$; similarly for $\pm h \in \Pi$ or $h \in \pm \Pi$. 
THEOREM 2. Suppose that the function $f$ satisfies the conditions of Theorem 1 , that is,

$$
\left\{\begin{array}{l}
\text { there exist positive auxiliary functions } a_{1}(\cdot), a(\cdot) \text { and some } \\
\text { constants } \gamma, c_{1}, c_{2} \in \mathbb{R} \text { with } c_{1} \neq-\alpha c_{2} \text { such that for } x>0 \\
\lim _{t \rightarrow \infty}\left(f(t x)-f(t)-a(t)\left(x^{\gamma}-1\right) / \gamma\right) / a_{1}(t) \\
=c_{1} \int_{1}^{x} s^{\gamma-1} \int_{1}^{s} u^{\alpha-1} d u d s+c_{2} \int_{1}^{x} s^{\alpha+\gamma-1} d s .
\end{array}\right.
$$

Then (2.10) is equivalent

(i) in case $\alpha=\gamma=0$ to: $\pm f(t)$ is second-order $\Pi$-varying (see [13]);

(ii) in case $\alpha=0, \gamma \neq 0$ to: $\pm t^{-\gamma} \tilde{f}(t) \in \Pi$ where

$$
\tilde{f}(t):= \begin{cases}f(t), & \gamma>0 \\ f(\infty)-f(t), & \gamma<0\end{cases}
$$

(iii) in case $\alpha<0$ to : there exists some constant c such that $\pm\left\{f(t)+c\left(t^{\gamma}-1\right) / \gamma\right\}$ satisfies $\left(1.2^{\prime}\right)$ with $\gamma$ replaced by $\alpha+\gamma$.

REMARKS 4. (i) It follows from the representations of the theorem that (2.10) holds locally uniformly in $(0, \infty)$ by the properties of the function classes to which the $f$ 's belong in the different cases (see, for example, $[1,8,15]$ ). The representations also lead to Potter bounds for relation (2.10) (cf. [1, Theorem 1.5.6] and [8, Propositions 1.7.5 and 1.19.4]).

(ii) Relation (2.10) implies that in all cases $a_{1}(t)=\mathrm{o}(a(t))$, as $t \rightarrow \infty$.

(iii) Note that in most cases the existence of a second-order relation makes the first order relation simpler; for example, in case $\alpha<0$ and $\alpha+\gamma=0$ one has $f(t) \sim c_{3} t^{\gamma}$, as $t \rightarrow \infty$.

PROOF OF THEOREM 2. The case $\gamma=\alpha=0$ is discussed in [13] so we restrict ourselves to the other cases.

(ii) Assume (2.10). By Theorem 1 the function $a(t)$ satisfies (2.2), that is, for $x>0$ we have

$$
a(t) \cdot x^{\gamma}=a(t x)-a_{1}(t) c_{1} x^{\gamma} \log x+o\left(a_{1}(t)\right), \quad t \rightarrow \infty .
$$

Substitution of this into (2.10) leads to

$$
\lim _{t \rightarrow \infty}\left\{\frac{\left[f(t x)-\gamma^{-1} a(t x)\right]-\left[f(t)-\gamma^{-1} a(t)\right]}{a_{1}(t)}\right\}=\left(c_{2}-\frac{c_{1}}{\gamma}\right) \frac{x^{\gamma}-1}{\gamma} .
$$

This relation is discussed in Proposition 2 and we obtain, for $\gamma>0$,

$$
f(t)-\gamma^{-1} a(t)=\gamma^{-2}\left(c_{2} \gamma-c_{1}\right) a_{1}(t)+\mathrm{o}\left(a_{1}(t)\right), \quad t \rightarrow \infty .
$$


Observe that this is also true in case $c_{2} \gamma-c_{1}=0$ by [1, Theorem 3.6.1] or [15, Theorem 2.11]. Furthermore using (2.2) and [8, Theorem 1.17], we know that there exists some $a_{2}(t) \sim a_{1}(t), t \rightarrow \infty$, such that

$$
t^{-\gamma} a(t)=c_{1} t^{-\gamma} a_{2}(t)+c_{1} \int_{1}^{t} s^{-\gamma-1} a_{2}(s) d s,
$$

and all together we end with

$$
\begin{aligned}
t^{-\gamma} f(t)= & \gamma^{-2}\left(c_{2} \gamma-c_{1}\right) t^{-\gamma} a_{1}(t)+\mathrm{o}\left(t^{-\gamma} a_{1}(t)\right)+c_{1} \gamma^{-1} t^{-\gamma} a_{2}(t) \\
& +c_{1} \gamma^{-1} \int_{1}^{t} s^{-\gamma-1} a_{2}(s) d s \\
= & c a_{3}(t) t^{-\gamma}+c_{1} \gamma^{-1} \int_{1}^{t} s^{-\gamma-1} a_{2}(s) d s,
\end{aligned}
$$

with a suitable function $a_{3}(t)$ satisfying $t^{-\gamma} a_{3}(t) \in R V_{0}, a_{3}(t) \sim a_{2}(t), t \rightarrow \infty$, and so we obtain by [8, Theorem 1.17] that $\pm t^{-\gamma} f(t) \in \Pi$ depending on the sign of $c_{1}$.

Conversely, if, for example, $t^{-\gamma} f(t) \in \Pi$, we have, with auxiliary function $\tilde{a}(t)=$ $t^{-\gamma} f(t)-t^{-1} \int_{0}^{t} s^{-\gamma} f(s) d s$, (see Thm 1.17 in [8]),

$$
\frac{(\lambda t)^{-\gamma} f(\lambda t)-\lambda^{-\gamma} f(\lambda)}{\tilde{a}(\lambda)} \longrightarrow \log t, \quad \lambda \rightarrow \infty,
$$

which is equivalent to

$$
\frac{f(\lambda t)-f(\lambda)-\gamma f(\lambda) \cdot\left(t^{\gamma}-1\right) / \gamma}{\lambda^{\gamma} \tilde{a}(\lambda)} \longrightarrow t^{\gamma} \log t, \quad \lambda \rightarrow \infty,
$$

which clearly is (2.10). The proof for the case $\gamma<0$ is similar.

(iii) By Proposition 2, relation (2.2) implies that $t^{-\gamma} a(t) \rightarrow c$ (say), and therefore $-t^{-\gamma} c_{1} a_{1}(t) / \alpha \sim c-t^{-\gamma} a(t), t \rightarrow \infty$. So we may choose $a_{1}(t)=\left(-\alpha t^{\gamma} / c_{1}\right)$ $\left(c-t^{-\gamma} a(t)\right)$. Substitution of $a(t)=c_{1} a_{1}(t) / \alpha+c t^{\gamma}$ into (2.10) yields for $x>0$ :

$$
\lim _{t \rightarrow \infty} \frac{\left(f(t x)-(c / \gamma)\left\{(t x)^{\gamma}-1\right\}\right)-\left(f(t)-(c / \gamma)\left\{t^{\gamma}-1\right\}\right)}{a_{1}(t)}=\left(\frac{c_{1}}{\alpha}+c_{2}\right) \frac{x^{\alpha+\gamma}-1}{\alpha+\gamma} \text {. }
$$

The inverse conclusion follows similarly as above by an easy calculation.

REMARKS 5. Considering instead of (1.5) the simple second-order relations

$$
\begin{array}{r}
\lim _{t \rightarrow \infty}\left\{\frac{f(t x)}{f(t)}-x^{\gamma}\right\} / A(t)=H(x), x>0, \text { in case } \gamma>0, \\
\lim _{t \rightarrow \infty}\left\{\frac{f(t x)-f(t)}{a(t)}-\log x\right\} / A(t)=H(x), x>0, \text { in case } \gamma=0, \\
\lim _{t \rightarrow \infty}\left\{\frac{f(\infty)-f(t x)}{f(\infty)-f(t)}-x^{\gamma}\right\} / A(t)=H(x), x>0, \text { in case } \gamma<0,
\end{array}
$$


(where the functions $H(x)$ denote different functions in the different cases) we get back the cases $\alpha=0$; or $\alpha<0$ and $\alpha+\gamma>0$; or $\alpha<0$ and $\gamma<0$; but not the cases $\alpha<0$ and $\gamma=0$; or $\alpha<0$ and $\gamma=-\alpha$; or $\alpha<0, \alpha+\gamma<0$ but $\gamma>0$.

\section{Inverse functions}

Next we consider the special case where $f$ is non-decreasing and give equivalent conditions in terms of $\phi:=f^{\leftarrow}$ (the inverse function of $f$ ). This is relevant for extreme-value statistics again, where $(2.10)$ is a condition in terms of the quantile function (so the inverse of a probability distribution) and one wants to have conditions in terms of the distribution function itself.

THEOREM 3. Suppose that $f$ is non-decreasing and $\phi$ is its right-continuous inverse function. Then (1.6) is equivalent to:

$$
\lim _{t / \gamma(\infty)}\left\{\frac{\frac{\phi(t+x \cdot a(\phi(t))}{\phi(t)}-(1+\gamma x)^{1 / \gamma}}{a_{1}(\phi(t)) / a(\phi(t))}\right\}=-(1+\gamma x)^{-1+1 / \gamma} H\left((1+\gamma x)^{1 / \gamma}\right)
$$

locally uniformly for $x \in(-1 / \max (0, \gamma), 1 / \max (-\gamma, 0))$.

REMARKS 6. (a) The result is also true for the left continuous inverse of $f$.

(b) In case $\gamma=0$ we define $(1+\gamma x)^{1 / \gamma}=e^{x}$.

(c) For specific parameters we can give more specific statements, such as, for example,:

(i) $\alpha=0, \gamma>0$; then $\pm t^{-1 / \gamma} \phi(t) \in \Pi$;

(ii) $\alpha=0, \gamma<0$; then $\pm t^{-1 / \gamma} \phi\left(f(\infty)-t^{-1}\right) \in \Pi$;

(iii) $\alpha<0, \gamma=0$; then

$$
\lim _{t \rightarrow \infty}\left(e^{-c(t+x)} \phi(t+x)-c\right) /\left(e^{-c t} \phi(t)-c\right)=e^{\alpha x}, x \in \mathbb{R} .
$$

PROOF. Since by Remark 4(i) relation (2.10) holds locally uniformly we can replace $x$ by $x(t)=1+\varepsilon a_{1}(t) / a(t)$ in $(2.10)$ and get:

(3.2) $\lim _{t \rightarrow \infty} \frac{f\left(t+t \varepsilon a_{1}(t) / a(t)\right)-f(t)-a(t)\left(\left(1+\varepsilon a_{1}(t) / a(t)\right)^{\gamma}-1\right) / \gamma}{a_{1}(t)}=0$,

hence

$$
\lim _{t \rightarrow \infty} \frac{f\left(t+t \varepsilon a_{1}(t) / a(t)\right)-f(t)}{a_{1}(t) / a(t)}=\varepsilon .
$$


Applying this for $\varepsilon>0$ and $\varepsilon<0$ and using $f(\phi(t)-) \leq t \leq f(\phi(t)+)$ we obtain $\lim _{t \rightarrow \infty}[f(\phi(t))-t] /\left[a_{1}(\phi(t)) / a(\phi(t))\right]=0$. This and (2.10) imply that

$$
\lim _{t \rightarrow \infty} \frac{f(\phi(t) x)-t-a(\phi(t))\left(x^{\gamma}-1\right) / \gamma}{a_{1}(\phi(t))}=H(x) .
$$

Now $F_{t}(x):=(f(\phi(t) x)-t) / a(\phi(t)), x>0, t>0$ is a family (with respect to t) of non-decreasing functions; furthermore, $\left(x^{\gamma}-1\right) / \gamma$ has a positive continuous derivative, the function $H(\cdot)$ is continuous and the function $a_{1}(\phi(t)) / a(\phi(t)) \rightarrow$ $0, t \rightarrow \infty$. Therefore we can apply an obvious generalisation of 'Vervaat's Lemma' (see [17, Lemma 1]) to deduce (3.1). The converse implication is similar.

EXAMPLE. Let $\Phi(t)=(2 \pi)^{-1 / 2} \int_{-\infty}^{t} e^{-y^{2} / 2}=d y$ be the standard normal probability distribution function and take $\phi(t):=1 /(1-\Phi(t))$. Since

$$
1-\Phi(t)=(2 \pi)^{-1 / 2} e^{-t^{2} / 2}\left(1 / t-1 / t^{3}+o\left(1 / t^{3}\right)\right)
$$

we find

$$
\lim _{t \rightarrow \infty} t^{2}\left(\frac{\phi(t+x / t)}{\phi(t)}-e^{x}\right)=\left(\frac{x^{2}}{2}+x\right) e^{x}
$$

for all real $x$, locally uniformly. We can apply Theorem 3 and find for $f$, the inverse function of $\phi$ :

$$
\lim _{t \rightarrow \infty}(f(t))^{3}\{f(t x)-f(t)-(\log x) / f(t)\}=-(\log x)^{2} / 2-\log x, \quad \text { for } x>0 .
$$

Hence, since $f(t) \sim \sqrt{2 \log t}(t \rightarrow \infty)$ and

$$
\sqrt{2 \log t}\{f(t)-\sqrt{2 \log t-\log \log t-\log 4 \pi}\} \rightarrow 0 \quad(t \rightarrow \infty),
$$

(see [10]), we find that for $x>0$

$\lim _{t \rightarrow \infty}(2 \log t)^{3 / 2}\left(f(t x)-f(t)-\frac{\log x}{(2 \log t-\log \log t-\log 4 \pi)^{1 / 2}}\right)=-\frac{(\log x)^{2}}{2}-\log x$.

Note that $f(t)$ is the quantile function of $\Phi$ at argument $(1-1 / t)$.

\section{Second-order variation and Laplace transforms}

We now consider the Laplace transforms of functions satisfying (1.6). Without loss of generality we assume $f \in L[0,1]$. Consider:

$$
\hat{f}(s)=\frac{1}{s} \int_{0}^{\infty} e^{-x / s} f(x) d x, \quad s>0 .
$$


Observe that, for any function satisfying (1.6), the abscissa of convergence is zero. We want to characterize second-order regular variation with the help of Laplace transforms for the case $\alpha+\gamma>-1$. Laplace transforms can be used for estimating the index of regular variation of the tail-function in a distribution with regularly varying tails. See, for example, the results in [6].

We begin with a first order result which is a mild extension of Karamata's Theorem on regular variation and Laplace transforms.

LEMMA 1. The following implications are true for an index $\gamma>-1$ :

(i) If $f$ satisfies the relation $\left(1.2^{\prime}\right)$ them $\hat{f}$ satisfies $\left(1.2^{\prime}\right)$.

(ii) If $f$ is non-decreasing and $\hat{f}$ satisfies $\left(1.2^{\prime}\right)$, then $f$ satisfies $\left(1.2^{\prime}\right)$.

(iii) $f$ satisfying $\left(1.2^{\prime}\right)$ implies

$$
\lim _{t \rightarrow \infty} \frac{f(t)-\hat{f}(t)}{f(t)-t^{-1} \int_{0}^{t} f(s) d s}=-\int_{0}^{\infty} e^{-s} \frac{s^{\gamma}-1}{\gamma} d s=-\frac{\Gamma(\gamma+1)-1}{\gamma} .
$$

PROOF. We define the auxiliary function

$$
g(t)=t f(t)-\int_{0}^{t} f(s) d s \quad\left(=\int_{0}^{t} s d f(s) \quad \text { if for example } f \text { is non-decreasing }\right) .
$$

Then (cf. [8, proof of Theorem 1.17]) $g \in R V_{\gamma+1}$ if and only if $f$ satisfies (1.2'). It is easy to see that for the Laplace transforms the following relation holds:

$$
\hat{f}(t)-\hat{f}(1)=\int_{1}^{t} \hat{g}(s) \frac{d s}{s^{2}} .
$$

From this relation one checks that $\hat{g} \in R V_{y+1}$ if and only if $\hat{f}$ satisfies $\left(1.2^{\prime}\right)$. Finally, if $f$ is nondecreasing, this is true for $g, \hat{f}$ and $\hat{g}$ as well.

(i) Using the Abelian part of Karamata's Theorem (see, for example, [1, Thm. 1.7.6]) and the remarks above we obtain: if $f$ satisfies $\left(1.2^{\prime}\right)$ with index $\gamma$, then $g \in R V_{\gamma+1}$ and hence $\hat{g} \in R V_{\gamma+1}$, which in turn implies $\hat{f}$ satisfies $\left(1.2^{\prime}\right)$.

(ii) If $\hat{f}$ satisfies $\left(1.2^{\prime}\right)$ with auxiliary function $a(t)$ then $\hat{g} \in R V_{y+1}$. Since $f$ nondecreasing implies $\hat{g}$ is non-decreasing, we find, by the Tauberian part of Karamata's Theorem, that $g \in R V_{y+1}$. The preliminary remarks of the proof now yield (ii).

(iii) This statement follows directly from [8, Theorems 1.20 and 1.8].

LEMMA 2. Suppose that $f$ satisfies $\left(1.2^{\prime}\right)$ with $\gamma=0$ and is bounded on $(0, a)$ for any $a>0$. Assume that $k:(0,1) \rightarrow \mathbb{R}$ is measurable and that for some $\varepsilon>0$ we have $t^{-\varepsilon} k(t)$ is integrable on $(0,1)$. Then

$$
\lim _{t \rightarrow \infty} \int_{0}^{1} k(s) \frac{f(t s)-f(t)}{a(t)} d s=\int_{0}^{1} k(s) \log s d s .
$$


PROOF. The assumption on $k$ implies (cf. [1, Lemma 4.1.1]) that there exists $\varepsilon>0$ such that

$$
\int_{0}^{t} k(s) d s=\mathrm{O}\left(t^{\varepsilon}\right) \quad \text { for } t \searrow 0 .
$$

The rest of the proof is similar to that of $[8$, Theorem 1.20].

Now we are able to state the main result in this section.

THEOREM 4. (a) (Abelian part) Assume that $f$ satisfies (2.10) with $\gamma \in \mathbb{R}$ and auxiliary functions $a(t) \in R V_{\gamma}, a_{1}(t) \in R V_{\alpha+\gamma}$ with parameters $\gamma>-1$ and $\alpha$ such that $\alpha+\gamma>-1$. Then the following relations hold:

$$
\lim _{t \rightarrow \infty} \frac{\hat{f}(t x)-\hat{f}(t)-\hat{a}(t)\left(x^{\gamma}-1\right) / \gamma}{a_{1}(t)}=\Gamma(\alpha+\gamma+1) \cdot H(x), \text { for } x>0
$$

and

$$
\lim _{t \rightarrow \infty} \frac{\hat{f}(t)-f(t)-a(t)(\Gamma(\gamma+1)-1) / \gamma}{a_{1}(t)}=\hat{H}(1)
$$

(In case $\gamma=0: \frac{\Gamma(\gamma+1)-1}{\gamma}:=\Gamma^{\prime}(1)=-\gamma_{0}$, where $\gamma_{0}$ is Euler's constant $)$

(b) (Tauberian part) Let be given some functions $a(t), a_{1}(t)$ such that the limit in (4.1) exists for all $x>0$ with $\gamma>-1$ and $H$ is not a multiple of $\left(x^{\gamma}-1\right) / \gamma$. Then $a_{1}(t)$ satisfies (2.3) with some $\alpha$ such that $\alpha+\gamma>-1$ and $\hat{a}(t)$ satisfies (2.2). Then $f$ is second-order regularly varying provided that the function

(i) $f \circ \exp$ is convex in case $\alpha=\gamma=0$;

(ii) $t^{-\gamma} \tilde{f}(t)$ is non-decreasing if $\alpha=0$ and $\gamma \neq 0$ ( $\tilde{f}$ is defined as in Theorem 2);

(iii) $f(t)+c\left(t^{\gamma}-1\right) / \gamma$ is non-decreasing if $\alpha<0$ (the constant $c$ is given by the representation in Theorem 2 ).

REMARKS 7. (i) Various parts of the Theorem are closely related to previous results, for example compare [7] for the case $\alpha=0, \gamma>0$ and compare [13] for the case $\alpha=\gamma=0$.

(ii) Other integral transforms, rather than Laplace transforms, in particular those with non-negative kernels and suitable behaviour at $\infty$ and 0 , can be considered as well. For example for case $\alpha+\gamma \leq-1$, one may use integral transforms based on the kernel $k(v)=e^{-v} v^{p}, p>0$ such that $p+\alpha+\gamma>-1$, in order to avoid problems at 0 . 
(iii) The Tauberian condition in part (b) on $f$ (monotonicity) may be weakened to one or two sided oscillation conditions as discussed for example in [1, Theorem 4.11.1].

(iv) One obtains in (4.2) (with $c_{1}, c_{2}$ as in $(2.10)$ and $\Gamma^{\prime}(1)=-\gamma_{0}$, where $\gamma_{0}=$ $0.577 \ldots$ is Euler's constant)

$$
\hat{H}(1)= \begin{cases}\left(\frac{c_{1}}{\alpha}+c_{2}\right) \frac{\Gamma(\alpha+\gamma+1)-1}{\alpha+\gamma}-c_{1} \frac{\Gamma(\gamma+1)-1}{\alpha \gamma}, & \text { if } \alpha<0, \gamma>0, \alpha+\gamma>-1 ; \\ \left(c_{2}-c_{1} / \gamma\right) \frac{\Gamma(\gamma+1)-1}{\gamma}+c_{1} \frac{\Gamma^{\prime}(\gamma+1)}{\gamma}, & \text { if } \alpha=0, \gamma>-1 \text { but } \gamma \neq 0 ; \\ \left(\frac{c_{1}}{\alpha}+c_{2}\right) \frac{\Gamma(\alpha+1)-1}{\alpha}-c_{1} \frac{\Gamma^{\prime}(1)}{\alpha}, & \text { if } \alpha \in(-1,0), \gamma=0 ; \\ \frac{c_{1}}{2} \Gamma^{\prime 2}(1)+c_{2} \Gamma^{\prime}(1), & \text { if } \alpha=\gamma=0 .\end{cases}
$$

PROOF OF THEOREM 4. For the case $\alpha=\gamma=0$ we refer to [13, Theorem 3.2]. Note that their Tauberian condition is satisfied if $f \circ \exp$ is convex. Furthermore the case $\gamma>0, \alpha=0$ is given in Theorem 1 in the paper of Geluk [7] under somewhat stronger conditions. The case $\alpha<0$ is a straightforward application of Lemma 1 (remember Theorem 2). So we only need to consider the case $\alpha=0, \gamma \neq 0$, where keeping Theorem 2 in mind: (a) follows directly from [8, Theorem 1.20] and Lemma 2. In the present application the kernel function is $k(t)=t^{\gamma} e^{-t}$; (b) follows directly from [8, Theorem 2.35], applied with the kernel $k(t)=t^{\gamma} e^{-t}$.

REMARK 8. In the paper [13] a Mercerian result is given for case $\alpha=\gamma=0$. (This is an implication from (4.2) to (2.10) under certain conditions.) Similar results could be given here in the other cases as well.

\section{References}

[1] N. H. Bingham, C. M. Goldie and J. L. Teugels, Regular variation, Encyclopedia Math. Appl. 27 (Cambridge University Press, Cambridge, 1987).

[2] R. Davis and S. Resnick, 'Tail estimates motivated by extreme value theory', Ann. Statist. 12 (1984), 1467-1487.

[3] A. Dekkers and L. de Haan, 'On the estimation of the extreme-value index and large quantile estimation', Ann. Statist. 17 (1989), 1795-1832.

[4] _ ' 'Optimal choice of a sample fraction in extreme-value estimation', J. Multivariate Anal. 47 (1993), 173-195.

[5] A. L. M. Dekker, On extreme-value estimation (Ph.D. Thesis. Erasmus University, Rotterdam, 1991). 
[6] W. Gawronski and U. Stadtmüller, 'Parameter estimations for distributions with regularly varying tails', Statist. Decisions 3 (1985), 297-316.

[7] J. L. Geluk, 'П-regular variation', Proc. Amer. Math. Soc. 82 (1981), 565-570.

[8] J. L. Geluk and L. de Haan, Regular variation, extensions and Tauberian theorems, CWI Tract 40 (CWI, Amsterdam, 1987).

[9] C. M. Goldie and R. L. Smith, 'Slow variation with remainder: Theory and applications', Quart. J. Math. Oxford Ser. 238 (1987), 45-71.

[10] L. de Haan, 'Equivalence classes of regularly varying functions', Stochastic Processes Appl. 2 (1974), 243-259.

[11] L. de Haan and S. Resnick, 'Second-order regular variation and rates of convergence in extremevalue theory', Ann. Probab. 24 (1996), 97-124.

[12] P. Hall, 'On some estimates of an exponent of regular variation', J. Roy. Statist. Soc. Ser. B 44 (1982), 37-42.

[13] E. Omey and E. Willekens, ' $\Pi$-variation with remainder', J. London Math. Soc. 37 (1988), 105118.

[14] T. T. Pereira, 'Second order behaviour of domains of attraction and the bias of generalized Pickands' estimator', in: Extreme value theory and applications III (eds. Galambos J., Lechner J., Simiu E.) NIST special publication 886 (1994) pp. 165-177.

[15] E. Seneta, Regularly varying functions, Lecture Notes in Math. 508 (Springer, Berlin, 1976).

[16] R. L. Smith, 'Uniform rates of convergence in extreme value theory', Adv. in Appl. Probab. 14 (1982), 600-622.

[17] W. Vervaat, 'Functional CLT's for processes with positive drift and their inverses', Z. Wahrsch. Verw: Gebiete 23 (1972), 245-253.

Erasmus University

Econometric Institute

P. O. Box 1738

3000 DR Rotterdam

The Netherlands
Universität Ulm

Abt. Math. III

89069 Ulm

Germany

e-mail: stamue@mathematik.uni-ulm.de 